\title{
Formação de mudas de alface provenientes de sementes peletizadas com altas diluições
}

\section{Lettuce seedlings formation from pelleted seeds with high dilutions}

\author{
${ }^{1}$ Roberto Luiz Queiroz, ${ }^{2}$ Elen Sonia Maria Duarte Rosa, ${ }^{3}$ Maximiliano Marques, ${ }^{3}$ Vanessa Aparecida \\ Goulart, ${ }^{3}$ Guilherme Ferreira Marques \\ ${ }^{1}$ Produção Agroecológica e Homeopatia, Centro Universitário do Sul de Minas (UNIS), Varginha, MG. \\ ${ }^{2}$ Departamento de Fitotecnia, IFET/BAIANO, Teixeira de Freitas, BA. \\ ${ }^{3}$ Curso de Engenharia Agronômica, Centro Universitário do Sul de Minas (UNIS), Varginha, MG. \\ Correspondência: robertoqueiroz@unis.edu.br
}

\section{Resumo}

A peletização de sementes vem sendo caracterizada como uma importante forma de maximização nos custos de produção de mudas pelo fato de reduzir o trabalho e mão de obra de distribuição manual de sementes, reduzindo ainda a prática do desbaste de plantas excedentes. No entanto, o interesse comercial pelo encapsulamento de semente avançou após a semeadura mecanizada, prática de plantio direto e, principalmente, pela possibilidade de inserção de nutrientes, reguladores de crescimento e aplicação de agrotóxicos como forma de constituir melhorias na sanidade das plantas. Nesse sentido, objetivou-se avaliar a produção de mudas de alface (Lactuca sativa L. c.v. Grand Rapids) oriundas de sementes peletizadas com preparados homeopáticos submetidas à cobertura de homeopatia de Phosphorus $30 \mathrm{CH}$. Os resultados apontam que a utilização de homeopatia de Sulphur $6 \mathrm{CH}$ e $\mathrm{Nux}$ vomica $6 \mathrm{CH}$ são capazes de propiciar mudas sadias e vigorosas quando comparadas com a testemunha.

Palavras-chave: agroecologia; homeopatia; Lactuca sativa L.

\begin{abstract}
The seed pelleting has been characterized as an important way of maximizing the seedlings production costs because of lower labor and manpower manual seed distribution, while reducing the practice of surplus plants thinning. However, the commercial interest in seed encapsulation advanced after mechanized seeding, tillage practice and especially the possibility of inclusion of nutrients, growth regulators and pesticides application as a way to provide improvements in the health of plants. In this sense, the objective was to evaluate the production of lettuce seedlings (Lactuca sativa L. cv. Grand Rapids) arising from pelleted seeds with homeopathic
\end{abstract}


preparations submitted homeopathy coverage Phosphorus $30 \mathrm{CH}$. The results show that the use of Sulphur $6 \mathrm{CH}$ homeopathy and $\mathrm{Nu} \times 6 \mathrm{CH}$ are able to provide healthy and vigorous seedlings when compared to the control.

Keywords: agroecology; homeopathy; Lactuca sativa $L$.

\section{Introdução}

A homeopatia vem se fundamentando como opção de tratamentos de seres abióticos e bióticos no intuito de reordenar a energia vital do organismo, bem como no equilíbrio de sintomas (Arruda et al., 2005). Desde a Instrução Normativa ํㅡ 007, de 17 de maio de 1999 e, mais recentemente por intermédio da Instrução Normativa no. 046, de outubro de 2011 (BRASIL, 2011), os preparados altamente diluídos (homeopatia) são recomendados tanto para o controle fitossanitário como para o reequilíbrio fisiológico das plantas de acordo com o enunciado do criador da Homeopatia, Samuel Hahnemann (17551843) e, ainda destacada como Tecnologia Social, conforme a UNESCO e a Fundação Banco do Brasil, em 2003 (Bonfim et al., 2012).

Em plantas, as experiências com uso da homeopatia, vêm sendo realizadas por agricultores nacionais e de outros países (Cuba, Inglaterra, Alemanha, França, Itália), com resultados surpreendentes na minimização de ataques de insetos, pragas (Almeida et al., 2003), germinação de sementes (Bonfim et al., 2012), aumento de compostos secundários (Duarte, 2007), fisiologia de plantas (Bonato, 2007) e produção de mudas sadias (Arruda et al., 2005).

Em sistemas vegetais hortícolas a ciência da ultradiluição é aplicável, pois os princípios fundamentais da homeopatia são coerentes com as leis da natureza, são imutáveis e pertinentes a todos os seres vivos. Casali, Castro e Andrade (2002), dissertam que a escolha do preparado homeopático a ser utilizado no vegetal deva ser pontuada pelas possibilidades de analogia com a matéria médica humana e animal; pelo "simillimum" do elemento químico nutricional de maior hierarquia na família, gênero, espécie ou variedade, e também por utilização da isopatia.

No aspecto medicamentoso e, de acordo com o conhecimento tradicional, Lactuca sativa é utilizada em diversos tratamentos, incluindo a insônia, tosse seca, dores reumáticas, ansiedade (Harsha e Anilakumar, 2012), também confere proteção contra estresse oxidativo, possivelmente pela atividade das vitaminas $\mathrm{C}$ e E, polifenóis e carotenoides (Harsha e Anilakumar, 2013). Além disso, o teor de nutrientes deste vegetal inclui quantidade apreciável de alguns minerais, como cálcio e ferro (Romani et al., 2002).

O negócio de hortaliças multiplicadas por sementes movimentou no varejo durante 2010/2011 a quantia de $R \$ 40,60$ bilhões. A alface contribui com $R \$ 7,79$ bilhões, representando a segunda principal hortaliça comercializada, sendo a alface tipo crespa com aproximadamente $53 \%$ do mercado nacional (Absem, 2011).

A prática de revestimento de sementes como forma de proteção de plantas é remota sendo originada no século XVIII onde há relatos de utilização de salmoura no controle de pragas (Costa, 2003). Atualmente, com avanço das doenças e pragas, a peletização de sementes tornou-se um fator importante para determinação do stand vegetativo comercial no campo, sendo utilizados diversos materiais tais como: fungicidas, nutrientes, 
pesticidas, polímeros, corantes de identificação dentre outros.

Diante das diversas limitações impostas pelos meios bióticos e abióticos no estabelecimento de plântulas e pela necessidade de desenvolver meios de sobrevivência do tegumento sem a utilização de insumos não permitidos em sistemas orgânicos de produção, objetivou-se verificar a influência de diversas peletizações homeopáticas no desenvolvimento inicial de alface.

\section{Material e Métodos}

O experimento foi realizado em condições de cultivo protegido na área de experimento do UNIS-MG, Varginha-MG. As sementes de alface, utilizadas no experimento, foram aquênios convencionais, da marca HORTICERES, cultivar GRAND RAPIDS, origem: E.U. A; porcentagem de germinação de $98 \%$ e pureza de $99 \%$.

O delineamento experimental foi no modelo em blocos ao acaso (DIC), em esquema fatorial $5 \times 3$, sendo quatro tratamentos homeopáticos além da testemunha - água destilada e dois tipos de cobertura de plantas: água destilada e homeopatia de Phosphorus $30 \mathrm{CH}$, perfazendo 15 tratamentos $\times 3$ repetições, em que cada parcela é representada por cinquenta células da bandeja, sendo os tratamentos discriminados como:

1. Sementes peletizadas/ Talco + Carbo vegetabilis $6 \mathrm{CH}$

2. Sementes peletizadas/ Talco $+\mathrm{Nux}$ vomica $6 \mathrm{CH}$

3. Sementes peletizadas/ Talco + Sulphur $6 \mathrm{CH}$

4. Sementes peletizadas/Talco + Arnica montana $6 \mathrm{CH}$

5. Sementes peletizadas/ Talco + água (testemunha)

6. Cobertura com Phosphorus $30 \mathrm{CH}$

7. Cobertura com água destilada
As sementes tratadas de alface foram plantadas em bandejas descartáveis de 200 células, preenchidas com substrato de fibra de coco sendo disposta a $1 \mathrm{~m}$ de altura do chão e irrigadas duas vezes ao dia.

A solução homeopática Phosphorus $30 \mathrm{CH}$ determinada como cobertura foi aplicada por aspersão (pulverizador manual tipo spray) nas folhas das mudas de alface após a semeadura com repetição a cada três dias, até o final do experimento. Utilizou-se um papel cartolina objetivando proteger os demais tratamentos da deriva do Phosphorus $30 \mathrm{CH}$ no momento da pulverização.

Os preparados homeopáticos foram adquiridos de farmácia de manipulação de medicamentos homeopáticos (Bioética Ltda. - Varginha-MG) em forma líquida (etanol 70\%). O processo de revestimento das sementes, ou seja, a peletização seguiu a metodologia de Bonfim e colaboradores (2012), respeitando a relação peso/volume, sendo: um grama de pó para um $\mathrm{mL}$ de adesivo. O insumo utilizado foi talco farmacêutico inerte, cuja composição é Mg3Si4O10(OH)2, e o adesivo refere-se aos tratamentos de soluções homeopáticas (Carbo Vegetabilis $6 \mathrm{CH}$, Nux Vomica $6 \mathrm{CH}$, Sulphur $6 \mathrm{CH}$ e Arnica $6 \mathrm{CH}$ ). Os preparados homeopáticos foram impregnados no talco farmacêutico com auxílio de placa de petri com intuito de formar um pó semi pastoso, íntegro, branco, o qual revestiu as sementes.

As características analisadas foram: Massa fresca (MFPA) e seca (MSPA) da parte aérea, Massa fresca (MFR) e seca (MSR) da raiz e Comprimento da Raiz (CR). A característica (CR) foi determinada no final do experimento, com auxílio de régua graduada sendo os resultados expressos em $\mathrm{mm}$. As características de massa fresca de parte aérea e raiz foram definidas, separadamente, por intermédio de pesagem em balança eletrônica de precisão de 0,001g. Para a obtenção das características de massa seca da parte aérea (MSPA) e massa seca de 
raiz (MSR), foi feita a embalagem (separados por tratamentos e características) em sacos de papel Kraft, encaminhado à secagem em estufa com ventilação forçada de ar, a $65^{\circ} \mathrm{C}$, até peso constante e determinada a massa seca em balança eletrônica de precisão de 0,01g.

Os dados foram submetidos à análise de variância e as médias foram comparadas pelo teste Tukey a $5 \%$ de probabilidade, no software ASSISTAT, versão 7.

\section{Resultados e Discussão}

De acordo com a análise de variância não se verificou interação entre peletização e cobertura para as características estudadas, permitindo a comparação/discussão dos fatores "homeopatia" e "cobertura", separadamente. Para o fator "homeopatia" somente a característica de massa seca de parte aérea (MSPA) e massa seca de raiz, para "cobertura" resultaram em efeitos não significativos.

As diferenças apontadas pela estatística (TABELA 1) sustentam a hipótese de que o talco e a água possuem a capacidade de receber a informação dos preparados homeopáticos e, previamente, em contato com outro biossistema retransmitir essa estrutura informacional (Bastide, 1995). Esses resultados apontam que os preparados homeopáticos foram capazes de provocar o sistema biológico da semente demonstrando a eficácia da homeopatia e contrapondo-se aos argumentos de efeito placebo, tão comumente propagada pelos que duvidam da ciência homeopática (TABELA 1).

TABELA 1. Resumo da análise de variância para massa fresca da parte aérea (MFPA), massa seca da parte aérea (MSPA), massa fresca da raiz (MFR), comprimento da raiz (CR) e massa seca da raiz (MSR), de mudas de alface c.v GRAND RAPIDS provenientes de peletização de sementes com homeopatias e submetidas à cobertura com preparado homeopático de Phosphorus 30CH. Varginha-MG, UNIS-MG, 2014.

\begin{tabular}{|c|c|c|c|c|c|c|}
\hline \multirow[b]{2}{*}{ F.V } & \multirow[b]{2}{*}{$\mathrm{GL}$} & \multicolumn{4}{|c|}{ QUADRADO MÉDIO } & \multirow[b]{2}{*}{$\begin{array}{c}\text { MSR } \\
(\mathrm{g})\end{array}$} \\
\hline & & $\begin{array}{c}\text { MFPA } \\
(\mathrm{g})\end{array}$ & $\begin{array}{c}\text { MSPA } \\
\text { (g) }\end{array}$ & $\begin{array}{c}\text { MFR } \\
(\mathrm{g})\end{array}$ & $\begin{array}{l}\mathrm{CR} \\
(\mathrm{cm})\end{array}$ & \\
\hline Peletização & 4 & $0,008599^{\star *}$ & $0,000028^{\text {ns }}$ & $0,009323^{\star}$ & $705,33^{*}$ & $0,000012^{* *}$ \\
\hline Cobertura & 1 & $0,179189^{*}$ & $0,000270^{*}$ & $0,050262^{*}$ & $1435,90^{*}$ & $0,000007^{\mathrm{ns}}$ \\
\hline Peletização*Cobertura & 4 & $0,001607^{\text {ns }}$ & $0,000101^{\mathrm{ns}}$ & $0,002880^{\mathrm{ns}}$ & $241,30^{\mathrm{ns}}$ & $0,000011^{\text {ns }}$ \\
\hline Resíduo & 109 & 0,002973 & 0,000055 & 0,001331 & 191,79 & 0,000005 \\
\hline CV (\%) & & 27,23 & 44,25 & 22,69 & 15,68 & 23,33 \\
\hline
\end{tabular}

${ }^{* *}$ Significativo pelo teste $\mathrm{F}$ a $5 \%$ de probabilidade.

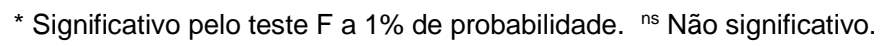

O comprimento de raiz foi a única característica afetada pela cobertura homeopática de Phosphorus $30 \mathrm{CH}$, resultando em resposta superior à média geral que foi de $88,33 \mathrm{~mm}$. Moraes (2009) obteve resposta no crescimento de raiz de eucalipto clone 3336 ao serem submetidos às dinamizações 6 e 12CH de Phosphorus. 
A homeopatia de fósforo foi capaz de estimular o metabolismo radicular das mudas de alface, demonstrando que essas plantas foram capazes de reagir à presença da homeopatia no substrato.

Em todas as homeopatias utilizadas observam-se respostas diferenciadas nas características analisadas, escapando a linearidade dos estímulos, apresentado pela significância nas diferenças das características analisadas, excetuando a MSPA, bem como, MSR com homeopatia Phosphorus $30 \mathrm{CH}$ em cobertura (TABELA 2). Esse padrão de resposta é explicado como revelação da relação particular do medicamento com o organismo (Silva, 2006), confirmando que as ultradiluições possuem frequência de onda própria (Bonato, 2007) podendo derivar na falta de relação proporcional entre o estímulo e a resposta (Bellavite, 2003).

Ao ser submetido à homeopatia de Sulphur $6 \mathrm{CH}$ as mudas de alface c.v GRAND RAPIDS produziu aumento de $0,0454 \mathrm{~g}$ planta ${ }^{-1}$ de massa fresca de parte aérea (MFPA) em relação à testemunha (água) que apresentou $0,1790 \mathrm{~g}_{\text {planta }}{ }^{-1}$ como o menor valor (TABELA 2).

TABELA 2: Massa fresca (MFPA) e seca (MSPA) de parte aérea, massa fresca (MFR) e seca (MSR) de raiz e comprimento da raiz (CR) de mudas de alface c.v GRAND RAPIDS, peletizadas com homeopatia expostas a cobertura de preparado homeopático de Phosphorus 30CH. Varginha-MG, UNIS-MG, 2014.

\begin{tabular}{|c|c|c|c|c|c|}
\hline \multirow{2}{*}{ Homeopatia } & \multicolumn{5}{|c|}{ Características analisadas } \\
\hline & MFPA (g) & MSPA (g) & MFR (g) & CR (mm) & MSR (g) \\
\hline Água (testemunha) & $0,1790 \mathrm{~b}$ & 0,0163 & $0,1621 a b$ & $88,56 a b$ & $0,0093 a b$ \\
\hline Carbo vegetabilis $6 \mathrm{CH}$ & $0,2137 \mathrm{ab}$ & 0,0181 & $0,1382 \mathrm{~b}$ & $79,95 \mathrm{~b}$ & $0,0082 \mathrm{~b}$ \\
\hline Sulphur $6 \mathrm{CH}$ & $0,2242 \mathrm{a}$ & 0,0159 & 0,1807 a & $94,30 \mathrm{a}$ & 0,0095 a \\
\hline Arnica montana $6 \mathrm{CH}$ & $0,1987 \mathrm{ab}$ & 0,0172 & $0,1400 \mathrm{~b}$ & $87,29 a b$ & $0,0086 a b$ \\
\hline Nux vomica $6 \mathrm{CH}$ & $0,1853 a b$ & 0,0152 & $0,1785 \mathrm{a}$ & 91,57 a & $0,0100 \mathrm{a}$ \\
\hline Cobertura com Homeopatia & MFPA (g) & MSPA (g) & MFR (g) & CR (mm) & MSR (g) \\
\hline Água (testemunha) & 0,2388 a & $0,0181 \mathrm{a}$ & $0,1798 \mathrm{a}$ & $84,87 \mathrm{~b}$ & 0,0089 \\
\hline Phosphorus 30CH & $0,1615 b$ & $0,0151 \mathrm{~b}$ & $0,1389 \mathrm{~b}$ & 91,79 a & 0,0093 \\
\hline CV(\%) & 27,23 & 44,25 & 22,69 & 15,68 & 23,33 \\
\hline Média Geral & 0,2002 & 0,0168 & 0,1607 & 88,33 & 0,0091 \\
\hline
\end{tabular}

Médias seguidas pela mesma letra na coluna não se diferem entre si estatisticamente pelo teste de Tukey a $5 \%$ de probabilidade.

Em estudos realizados por Bonato e Silva (2003) ficou comprovado a influência do preparado homeopático Sulphur $5 \mathrm{CH}$ no aumento da produção de matéria seca da parte aérea de rabanete, diferentemente deste experimento onde a mesma característica (massa seca) não diferiu estatisticamente entre os tratamentos. Resultados semelhantes foram apresentados por Bonato, Proença e Reis (2009) que ao utilizar homeopatia de Sulphur $6 \mathrm{CH}$ obtiveram incrementos na massa fresca de plantas de Mentha arvensis, bem como o aumento no conteúdo de óleo essencial. 
Nas características inerentes à raiz (MFR, CR e MSR) nota-se que a homeopatia de Carbo vegetabilis $6 \mathrm{CH}$ resultou em redução significativa comparativamente ao preparado homeopático de Sulphur $6 \mathrm{CH}$ que proporcionou as melhores médias no sistema radicular das mudas de alface. Esse resultado é semelhante à pesquisa de Pulido e colaboradores (2014) que obtiveram o mesmo padrão estatístico, ou seja, melhores resultados utilizando Sulphur $6 \mathrm{CH}$ e piores usando ultradiluição de Carbo vegetabilis $6 \mathrm{CH}$ nas variáveis: matéria seca de raiz e longitude da maior raiz de plântulas de repolho híbrido Fuyutoy.

Os aspectos estatísticos na resposta de Nux vomica $6 \mathrm{CH}$ nas características da raiz de mudas de alface c.v. GRAND RAPIDS, assemelharam-se ao tratamento com Sulphur $6 \mathrm{CH}$. Moreno (2000) e Brunini e Sampaio (1994) caracterizam as homeopatias de Sulphur e Nux vomica como os principais policrestos da matéria médica, cuja patogenesia é extensa e com os mais diversificados sintomas. Efeito constatado em plantas de alface c.v. Regina por Queiroz (2015), que comprovou aumento nas médias das características MFF (massa fresca das folhas), NF (número de folhas) e MSF (massa seca das folhas) submetidas ao preparado de Nux vomica $6 \mathrm{CH}$ quando comparado ao tratamento controle (água destilada).

Preparado homeopático de Sulphur manifesta-se fortificando a reação orgânica e "jogando fora" aquilo que se enquadra como prejudicial à sua vitalidade. Sendo indicado em casos de intoxicação, que se externaliza na superfície do corpo, bem como em supressões que causam sintomas variáveis e alternantes, dadas as alterações do meio (Voisin, 1987). Por outro lado, a ultradiluição de Nux vomica, tem sido maximizada como grande descontaminador de plantas intoxicadas por agrotóxico e por adubos químicos sintéticos solúveis, agindo nos tecidos das plantas e também no solo, propiciando-lhes a homeostase (Cupertino, 2008).

Possivelmente as respostas significativas das ultradiluições de Nux vomica e Sulphur possam ter contribuído para com toda a potencialidade reação aos fatores externos cumprindo com a busca inerente pela sobrevivência e pela auto regulação (Andrade, 2004) das sementes de alface c.v. GRAND RAPIDS que estavam tratadas de forma convencional (inseticida e fungicida), antes da peletização com preparados homeopáticos.

As respostas positivas das ações homeopáticas condizem com a teoria de Schembri (1992) e Vithoulkas (1980). Os pesquisadores investigaram os preparados homeopáticos em baixas dinamizações, que por serem densos e moleculares, são mais eficazes no corpo físico como no caso dos vegetais.

Arenales (1999) evidencia que o Carbo vegetabilis é o medicamento da geada, pois a geada queima os tecidos vegetais pelo frio, assim como o fogo queima o vegetal pela ação do calor. Com relação às exigências de clima, as plantas de alface têm preferência por climas amenos, tolerando geadas leves. Por esse aspecto a preparação homeopática Carbo vegetabilis não possui similitude com mudas de alface, podendo-se inferir que as respostas negativas de Carbo vegetabilis $6 \mathrm{CH}$ nas características de raiz de mudas de alface c.v. GRAND RAPIDS, são sinais artificiais (patogenesia), denominada segundo Kent (1996) de ação primária do ser submetido aos estímulos de preparados ultradiluídos.

\section{Conclusões}

A cobertura com homeopatia de Phosphorus $30 \mathrm{CH}$, propiciou maior comprimento de raiz, quando comparada com aplicação de água (testemunha). 
As homeopatias de Nux vomica $6 \mathrm{CH}$ e Sulphur $6 \mathrm{CH}$, promoveram incrementos nas características condicionadas à raiz de mudas de alface c.v. GRAND RAPIDS, quando confrontada com o tratamento Carbo vegetabilis $6 \mathrm{CH}$.

Todos os medicamentos homeopáticos foram capazes de provocar maiores efeitos positivos e incrementaram, a depender do tratamento, a massa fresca de parte aérea.

As preparações não moleculares de Carbo vegetabilis $6 \mathrm{CH}$ reduziu, significativamente, as características de raiz analisadas.

\section{Referências}

ASSOCIAÇÃO BRASILEIRA DO COMÉRCIO DE SEMENTES E MUDAS - ABSEM. 2014 Projeto para o levantamento dos dados socioeconômicos da cadeia produtiva de hortaliças no Brasil, 2010/2011. Campinas, $2011 . \quad$ Disponível: http://www.abcsem.com.br/docs/direitos_reservados .pdf. Acesso em: 12 jan 2015.

ALMEIDA, A. A. de; GALVÃO, J. C. C.; CASALI, V. W. D.; LIMA, E. R. de; MIRANDA, G. V. 2003. Tratamentos homeopáticos e densidade populacional de Spodoptera frugiperda (J.E.Smith, 1797) (Lepidoptera: Noctuidae) em plantas de milho no campo. Revista Brasileira de Milho e Sorgo, Sete Lagoas, v.2, n.2, p.1-8.

ANDRADE, F. M. C. 2004. Alterações da vitalidade do solo com o uso de preparados homeopáticos. 378p. Tese (Doutorado em Fitotecnia), Universidade Federal de Viçosa, Viçosa, 2004.

ARENALES MC. 1999. A homeopatia na agropecuária orgânica. In: Encontro Mineiro sobre Produção Orgânica de Hortaliças. 1, 1998. Viçosa, MG. ANAIS... UFV, 1999. p.54-6.
ARRUDA, V. M.; CUPERTINO, M. do C.; LISBOA, S. P.; CASALI, V. W. D. 2005. Homeopatia tri-uma na agronomia. UFV: Viçosa, $119 \mathrm{p}$.

BASTIDE, M. Basic research on high dilution effects. In: TADDEI-FERRETTI, C.; MAROTTA, P. (Ed.). 1995. High dilution effects on cells and integrated systems. London: Words Scientific Publishing, v. 3, p. 3-15.

BELLAVITE, P. 2003. Complexity science and Homeopathy: a synthetic overview. Homeopathy, London, v. 92, p.203-212.

BONATO, C. M.; SILVA, E. P. 2003. Effect of the homeopathic solution Sulphur on the growth and productivity of radish. Acta Scientiarum, v. 25, p. 259-263.

BONATO, C. M. 2007. Homeopathy in the host physiology. Fitopatologia Brasileira, v. 32, supl., p. 79-82.

BONATO, C. M.; PROENÇA, G.T.; REIS, B. 2009 Homeopathic drugs Arsenicum album and

Sulphur affects the growth and essential oil content in mint (Mentha arvensis). Acta Scientiarum, v. 31, p. 101-105.

BONFIM, F. P. G.; CASALI, V. W. D.; MENDONCA, E. G.; MARTINS, E. R. 2012. Estresse hídrico em feijoeiro (Phaseolus vulgares L.) tratado com os preparados homeopáticos de Arnica montana. Enciclopédia biosfera, v. 8, p. 530- 538.

BRASIL. 2011. Ministério da Agricultura, Pecuária e Abastecimento. Instrução Normativa n. 46 de 6 de outubro de 2011. Estabelecer o Regulamento Técnico para os Sistemas Orgânicos de Produção Animal e Vegetal, bem como as listas de Substâncias Permitidas para uso nos Sistemas Orgânicos de Produção Animal e Vegetal. Diário Oficial [da] República Federativa do Brasil. Brasília, DF. 
BRUNINI, C.; SAMPAIO, C. 1994. Homeopatia: princípios, doutrina e farmácia. São Paulo: Mythos Editora, $247 \mathrm{p}$.

CASALI, V.W.D.; CASTRO, D.M.; ANDRADE, F.M.C. 2002. Pesquisa sobre homeopatias nas plantas. In: Seminário Brasileiro Sobre Homeopatia na Agropecuária Orgânica, 3ํㅡㄹ Campinas do Sul - RS, Anais... p. 16-24.

COSTA, M. de A. 2003. Peletização de sementes de brócolos em leito de jorro cônico. 243p. Tese (Doutorado em Engenharia Química). Universidade Estadual de Campinas, Campinas.

CUPERTINO, M.C. 2008. O conhecimento e a prática sobre homeopatia pela família agrícola. 132p. Dissertação (Mestrado em Fitotecnia), Universidade Federal de Viçosa.

DUARTE, E.S.M. 2007. Crescimento e teor de óleo essencial em plantas de Eucalyptus citriodora e Eucalyptus globulus tratadas com homeopatia. 188p. Tese (Doutorado em Fitotecnia), Universidade Federal de Viçosa, Viçosa.

KENT, J. T. 1996. Filosofia homeopática. Tradução: Ruth Kelson. São Paulo: Robe.

HARSHA, S. N.; ANILAKUMAR, K. R. 2012. Effects of Lactuca sativa extract on exploratory behavior pattern, locomotor activity and anxiety in mice. Asian Pacific Journal Tropic, v. 47, p.9 - 15.

HARSHA, S. N.; ANILAKUMAR, K. R. 2013. Antioxidant properties of Lactuca sativa leaf extract involved in the protection of biomolecules. Biomedicine \& Preventive Nutrition. v. 3, p. 367-373.
MORAES, L.C.C.A. de V. 2009. Crescimento e qualidade de mudas clonais de eucalipto com aplicação de preparados homeopáticos. 54f. Tese (Mestrado em Fitotecnia) - Universidade Federal de Viçosa, Viçosa.

MORENO, J. A. 2000. Organon a arte de curar de Samuel Hahnemann. Belo Horizonte: Hipocrática Hahnemanniana, $277 \mathrm{p}$.

PULIDO, E.E.; BOFF, P.; DUARTE, T.S.; BOFF M. 2014. Preparados homeopáticos en el crecimiento y en la producción de repollo cultivado en sistema orgánico. Horticultura Brasileira, v. 8, p. 267-272.

QUEIROZ, R.L. 2015. Alelopatia de manjericão e uso do preparado homeopático Nux vomica em alface. 78f. Tese (Doutorado em Agronomia) - Universidade Estadual Paulista, Botucatu.

ROMANI, A.; PINELLI, P.; GALARDI, C.; SANI, G.; CIMATO, A.; HEIMLER, D. 2002. Polyphenols in greenhouse and open-air-grown lettuce. Food Chem. v. 79, p. $337-342$.

SCHEMBRI, J. 1992. Conheça a Homeopatia. 3 ed. Belo Horizonte: Rona, 268 p.

SILVA, R. T. B. da. 2006. Interpretação matemáticofísica dos efeitos de ultradiluições em Sphagneticola trilobata (L.) Pruski. 140f. Tese (Doutorado em Fitotecnia) - Universidade Federal de Viçosa, Viçosa.

VITHOULKAS, G. 1980. Homeopatia: ciência e cura. Tradução: Sônia Régis. São Paulo: Cultrix, 436 p.

VOISIN, H. 1987. Manual de matéria médica para o clí́nico homeopata. 2 ed. São Paulo: Andrey, 1160 p. 